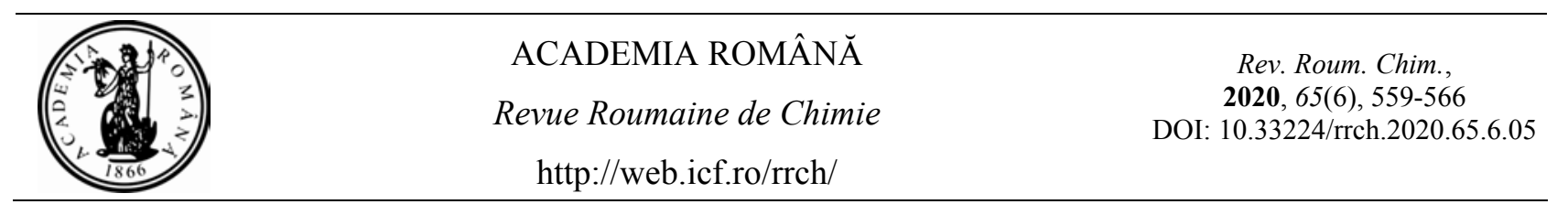

\title{
SURFACE PASSIVATION OF CARBON NANOPARTICLES WITH 1,2-PHENYLENEDIAMINE TOWARDS PHOTOLUMINESCENT CARBON DOTS
}

\author{
Andreea P. CRISAN, ${ }^{a}$ Ana-Maria CRACIUN, ${ }^{\mathrm{b}}$ Iuliana MIHALACHE, ${ }^{\mathrm{c}}$ Monica FOCSAN, ${ }^{\mathrm{b}}$ \\ Crina SOCACI, ${ }^{\mathrm{a}, \mathrm{d}}$ Dana MANIU, ${ }^{\mathrm{b}}$ Simion ASTILEAN, ${ }^{\mathrm{b}, *}$ L. Monica VECA ${ }^{\mathrm{c}, *}$ and Anamaria TEREC ${ }^{\mathrm{A}, *}$ \\ ${ }^{a}$ Faculty of Chemistry and Chemical Engineering, Babes-Bolyai University, Cluj-Napoca 400084, Roumania \\ ${ }^{\mathrm{b}}$ Laser Microspectrocopy and Nanobiophotonics Center, Interdisciplinary Research Institute in Bio-Nano-Sciences \\ and Faculty of Physics, Babes-Bolyai University, 1 M. Kogalniceanu, 400084, Cluj-Napoca, Roumania \\ ${ }^{c}$ National Institute for Research and Development in Microtechnologies, IMT-Bucharest, Bucharest 077190, Roumania \\ ${ }^{\mathrm{d}}$ National Institute of Research and Development for Isotopic and Molecular Technologies, Donath 67-103, \\ RO-400293 Cluj-Napoca, Roumania
}

The chemistry of carbon dots (CDs) surface has enabled the development of new luminescent nanomaterials with wide and precise array of applications. In respect to this, we perform the surface passivation of carboxylated carbon nanoparticles with the aromatic 1,2-phenylenediamine to prepare water-soluble photoluminescent CDs
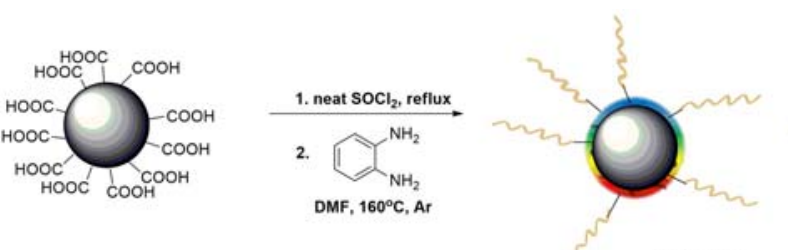

$\sim \sim$ = benzimidazole-

o-PD-CDs exhibiting a photoluminescence (PL) quantum yield of $8 \%$ at $575 \mathrm{~nm}$, a composite photoluminescence signal in the $330-500 \mathrm{~nm}$ domain, and an average lifetime of 1.93 ns. This new synthetic strategy promoted the formation of carbon dots covered with benzimidazole-containing oligomers of 1,2-phenylenediamine (o-PD) that, by taking advantage of the benzimidazole derivatives' biological activity and their application in Material Science, could lead to various new applications.

\section{INTRODUCTION}

Among luminescent materials, the latest member of the carbonaceous family, carbon dots (CDs), with their multicolor behavior, ${ }^{1,2}$ became an attractive nanomaterial due to their photophysical properties which render them vast applicability, such as solid-state lighting, sensing, and multiplexed biological labeling. While carbon dots and traditional semiconductor quantum dots (QDs) show similar chemical stability and high photoluminescence quantum yield, carbon dots are the less toxic, non-photobleaching and lower-cost alternative. Unlike conventional QDs and organic dyes, CDs also exhibit broad emission dependent upon the excitation wavelength and longer decay lifetimes, which make them suitable for bioimaging and optoelectronic applications.

One of the most common theories used to explain the PL mechanism is the surface state-controlled radiative recombination. Even though the PL mechanism has yet to be fully understood, there is experimental evidence that the optical properties are highly sensitive to the carbon dots synthetic pathway,

\footnotetext{
*Corresponding author: simion.astilean@phys.ubbcluj.ro, asuciu@chem.ubbcluj.ro,monica.veca@imt.ro
} 
the type of starting materials, more precisely they are generally strongly dependent on the efficiency of the surface passivation.

The passivation is often achieved with oligomers or small organic molecules containing heteroatoms. It has been proven that the heteroatom has a dual role: passivation and doping the CDs surface to yield CDs with improved photoluminescence quantum yield and longer decay lifetime. Passivation with polymers like polyethylene glycol (PEG), amine terminated polyethylene glycols (PEG-1500N ${ }^{5}$, PEG-200N ${ }^{6}$ ), poly(propionylethyleneimine-coethyleneimine) (PPEI-EI) ${ }^{6}, \quad 4,7,10$-trioxa-1,13tridecanediamine (TTDDA) ${ }^{7}$ and polyethyleneimine $(\mathrm{PEI})^{8}$ was the most investigated method for enhancing the PL of CDs.

Among passivation methods, covalent linkage of diamines containing molecules, such as 1,2-ethylenediamine $^{9}$, 1,4-butanediamine ${ }^{4}, 2,2^{\prime}$-(ethylenedioxy)bis(ethylamine) ${ }^{10}$, diethylamine ${ }^{11}$ is frequently used, due to their accessibility and effective way to improve the carbon dots' optical properties. Most of the studies focused on aliphatic amines with few reports using aromatic diamine as a surface passivation moiety. For example, the CDs passivated with $p$-phenylenediamine ( $p$-PD) have shown a stable excitation independent green photoluminescence with a quantum yield of $14 \%$ and lifetime of 11.8 ns. ${ }^{12}$ As observed for CDs synthesized from aromatic diamine in the microwave assisted methods ${ }^{13,14}$ it is expected to see a red shift in the emission maximum with increasing the content of $\mathrm{N}$ and $\mathrm{C}=\mathrm{N}$ or $\mathrm{C}=\mathrm{O} /$ $\mathrm{CONH} .{ }^{15}$

Aiming at extending the current blue-green photoluminescence emission of carbon dots to lower energies, in this work, we have synthesized photoluminescent CDs coated with benzimidazolecontaining oligomers employing a condensation reaction between $o$-phenylenediamine $(o-\mathrm{PD})$ and carboxylated carbon nanoparticles. The surface functionalization of carboxylated carbon nanoparticles with aromatic amines has been scarcely studied so $\operatorname{far}^{12}$ and our findings regarding the formation of benzimidazole are of great synthetic interest due to benzimidazole derivatives' known biological activity ${ }^{16}$ and efficiency as electron-transporter. ${ }^{17}$

\section{RESULTS AND DISSCUSION}

\section{Synthesis and structural characterization}

The newly functionalized CDs were obtained by a multi-step synthetic strategy (Scheme 1) that included oxidation of bare carbon nanoparticles to introduce carboxyl groups at the surface as well as separation of the smallest particles. The latter were reacted with thionyl chloride, followed, after removal of excess thionyl chloride, by amidation with $o-\mathrm{PD}$ in the presence of $\mathrm{N}, \mathrm{N}-$ dimethylformamide to access $o$-PD-CDs.

A control sample, named $o-\mathrm{PD}_{0}$, was obtained by heating $o-\mathrm{PD}$ in $\mathrm{DMF}$ at $160^{\circ} \mathrm{C}$, without addition of carbon nanoparticles and their properties compared with those of $o$-PD-CDs.

${ }^{1} \mathrm{H}$ - and ${ }^{13} \mathrm{C}-\mathrm{NMR}$ spectroscopy were firstly employed for characterization of the passivating shell of $o$-PD-CDs. The ${ }^{1} \mathrm{H}-\mathrm{NMR}$ spectrum recorded for the suspension of $o$-PD-CDs in $\mathrm{D}_{2} \mathrm{O}$ (Figure 1a) is very simple, showing two doublets of doublets at $7.33 \mathrm{ppm}$ and $7.68 \mathrm{ppm}$ and one singlet $(8.22 \mathrm{ppm})$, and is very similar to the spectrum recorded for the suspension of $o-\mathrm{PD}_{0}$ in $\mathrm{D}_{2} \mathrm{O}$. The pattern and chemical shifts are specific for benzimidazole. The cyclization reaction of $o$ PD has been previously reported to yield benzimidazole when heated under microwaves ${ }^{18}$ or in the presence of acidic catalyst. ${ }^{19}$ The ${ }^{1} \mathrm{H}$ NMR data are well correlated with the ${ }^{13} \mathrm{C}$-NMR spectrum (Figure 1b) where signals for carbon atoms in benzimidazole appear. As a result, we concluded that $o$-PD has reacted with the N,Ndimethylformamide used as solvent to obtain benzimidazole, soluble in the deuterated solvent. As bare benzimidazole exhibits no fluorescence, while our material has strong fluorescence, we suppose that further reaction with $o$-PD occurred in both $o-\mathrm{PD}_{0}$ and $o$-PD-CDs, with further attachment by amidation with carbonyl chloride-decorated surface of the CDs in the case of the latter and that the $o$-PD-CDs are actually covered with benzimidazole-containing oligomers of $o$-PD.

The FT-IR technique was employed to identify the organic functional groups on the surface of the functionalized CDs (Figure 2) and support our supposition. The FT-IR spectrum of $o$-PD-CDs revealed some structural information and confirmed the functionalization of CDs.

For example, the presence in the $o$-PD-CDs IR spectrum of specific $\mathrm{C}=\mathrm{O}$ and $\mathrm{C}=\mathrm{N}$ stretching vibrations at $1770 \mathrm{~cm}^{-1}$ and $1619 \mathrm{~cm}^{-1}$ respectively, indicates once again the formation of new nitrogencontaining synthons during $o$-PD-CDs formation, ${ }^{20,21}$ correlated also with literature data on IR spectra for benzimidazole derivatives showing the presence of a band at around $1620 \mathrm{~cm}^{-1}$. 22 Except for the abovementioned band, the FT-IR spectrum of the control product $\left(o-\mathrm{PD}_{0}\right)$ presents a similar pattern, but a lower intensity of most peaks. 

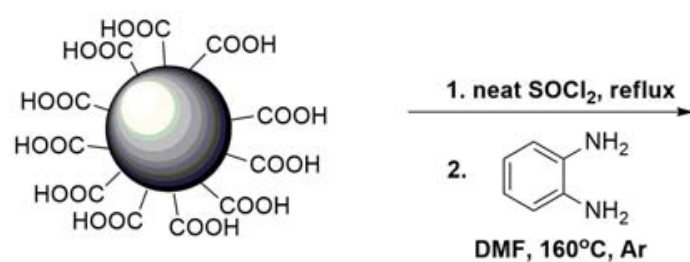

$\mathrm{DMF}, 160^{\circ} \mathrm{C}, \mathrm{Ar}$

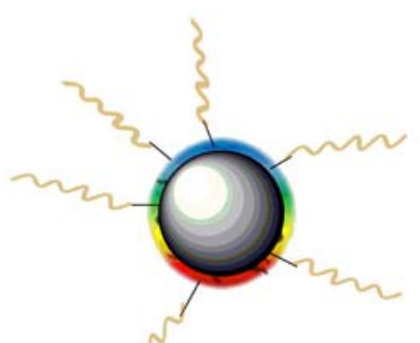

o-PD-CDs
= benzimidazole-

containing oligomers

Scheme 1 - Schematic representation for the preparation of $o$-PD-CDs.

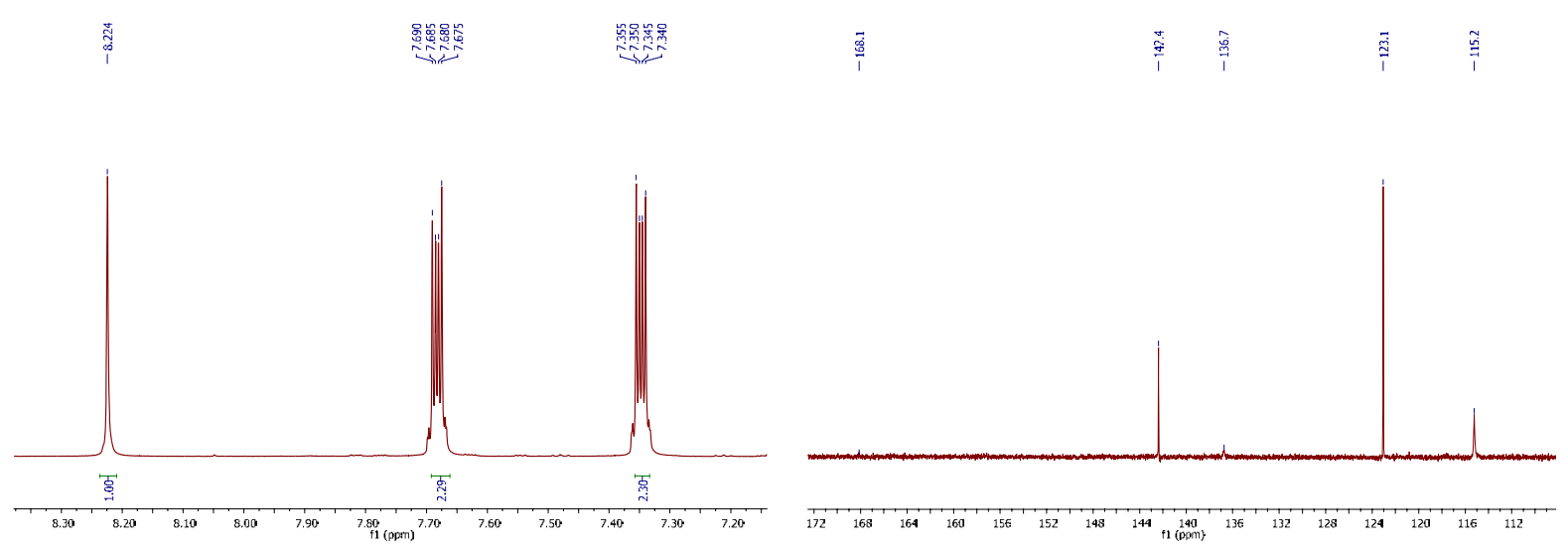

Fig. 1 - Fragments of a) ${ }^{1} \mathrm{H}$ NMR $\left(600 \mathrm{MHz}, \mathrm{D}_{2} \mathrm{O}\right)$ (left) and b) ${ }^{13} \mathrm{C} \mathrm{NMR}\left(150 \mathrm{MHz}, \mathrm{D}_{2} \mathrm{O}\right)$ (right) spectra of $o$-PD-CDs.

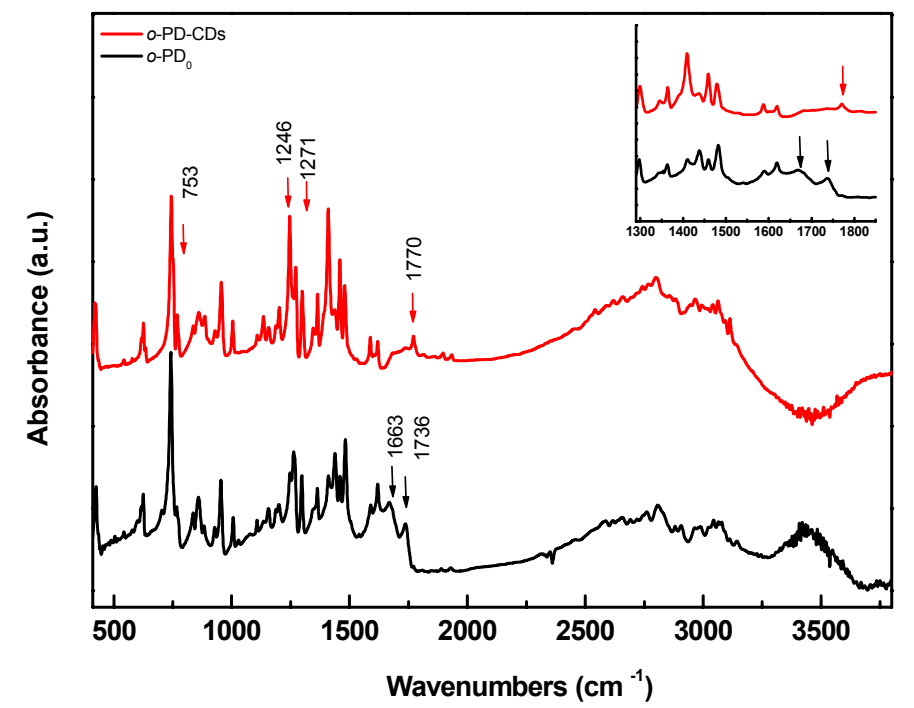

Fig. 2 - FT-IR spectra of $o$-PD-CDs and $o-\mathrm{PD}_{0}$. Inset: focus on $1300-1800 \mathrm{~nm}$ region.

\section{Optical characterization}

The UV-Vis absorption spectra of the obtained functionalized CDs are presented in Figure 3, revealing different optical absorption features.

The absorption spectrum of $o$-PD-CDs is dominated by intense high-energy absorption bands located in the $240-280 \mathrm{~nm}$ spectral region. Specifically, $o$-PD-CDs display three significant absorption bands at 245, 270 and $278 \mathrm{~nm}$, which can be assigned to the $\pi-\pi^{*}$ electron transition of nitrogen-conjugated $\mathrm{C}=\mathrm{C}$ bonds ${ }^{23}$ from the benzimidazole-containing oligomers of $o$-PD covering the CDs, as we have previously concluded from the structural studies. The weak band around $300 \mathrm{~nm}$ can be assigned to the $\mathrm{n}-\pi^{*}$ electron transition of $\mathrm{C}=\mathrm{O}$ related to amide bonds. ${ }^{24}$ The lower-energy absorption band around 
$415 \mathrm{~nm}$ can probably be assigned to polaron- $\pi^{*}$ transitions from $\mathrm{CDs}^{21}$ or to the $\pi-\pi^{*}$ transition in $\mathrm{C}=\mathrm{N}$. The spectrum of $o-\mathrm{PD}_{0}$ shares similar features to the absorption spectra of $o$-PD-CDs (see inset Figure 3), in the UV region, however in the visible region the sample does not exhibit any absorption band.

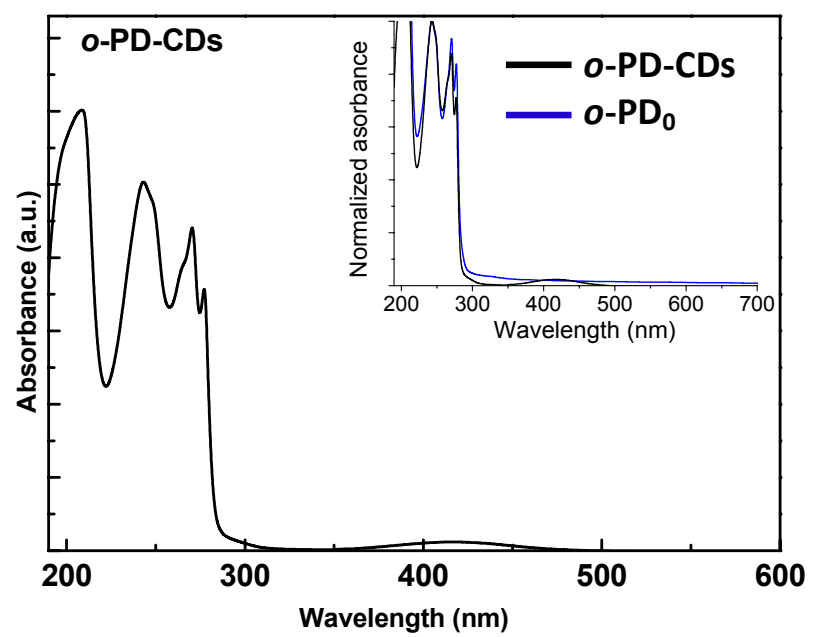

Fig. 3 - Absorption spectrum of the as-prepared $o$-PD-CDs. Inset: Comparison with the absorption spectrum of reference sample $o-\mathrm{PD}_{0}$.

(a)

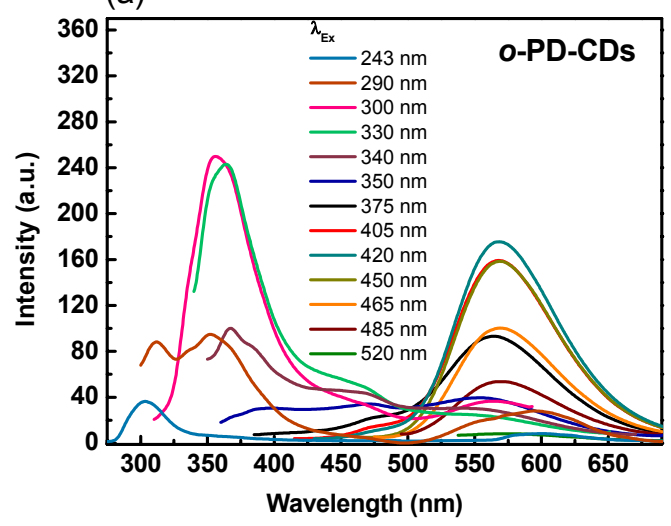

(b)

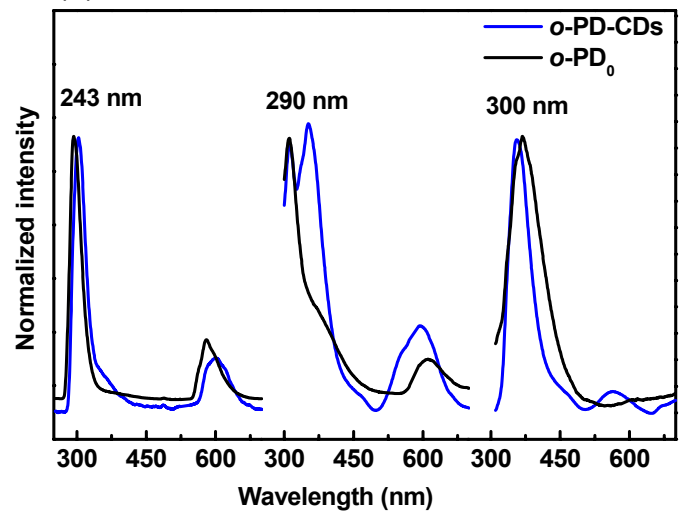

Fig. 4 - PL spectra of amine-functionalized CDs recorded at progressively longer excitation wavelength (a) and comparison with the emission of $o-\mathrm{PD}_{0}$ at selected excitation wavelengths: $243 \mathrm{~nm}, 290 \mathrm{~nm}$ and $300 \mathrm{~nm}$ (b).

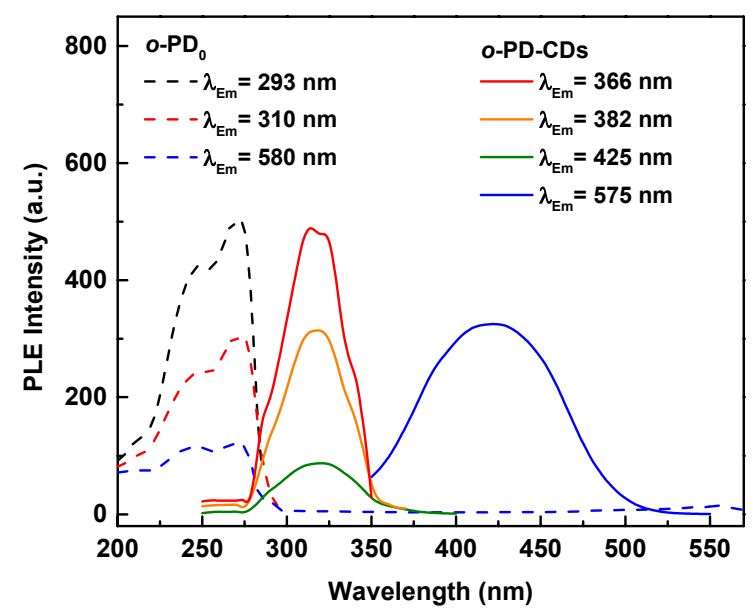

Fig. 5 - Comparison between the excitation spectra of $o-\mathrm{PD}-\mathrm{CDs}$ and $o-\mathrm{PD}_{0}$ at selected emission wavelengths. 


\section{Steady state and time - resolved fluorescence measurements}

To evaluate the effect of surface passivation on the optical properties of the $o$-PD-CDs, we carried out a detailed PL study using different excitation wavelengths. As shown in Figure 4(a), the obtained $o$-PD-CDs samples exhibit some interesting PL properties. We observe a composite PL signal in the $330-550 \mathrm{~nm}$ region, shifting from $303 \mathrm{~nm}$ to $400 \mathrm{~nm}$ when increasing the excitation wavelength from $250 \mathrm{~nm}$ to $350 \mathrm{~nm}$, simultaneously with a weaker PL in the $500-600 \mathrm{~nm}$ region for excitations above $310 \mathrm{~nm}$. The presence of the PL band, located at $575 \mathrm{~nm}$, is clearly distinguished for excitation between $330 \mathrm{~nm}$ and $520 \mathrm{~nm}$, with the highest intensity at $420 \mathrm{~nm}$ excitation.

In order to better understand the origin of these intriguing PL properties displayed by $o$-PD-CDs we also monitored the emissive properties of the control sample, $o-\mathrm{PD}_{0}$, resulted from similar synthetic procedures carried out in the absence of carbon nanoparticles. For excitation between 250 $\mathrm{nm}$ and $290 \mathrm{~nm}$, the sample exhibits an intense emission band at 349, 366, $385 \mathrm{~nm}$ and a weaker one at around $549 \mathrm{~nm}$. However, beyond $370 \mathrm{~nm}$ excitation, $o-\mathrm{PD}_{0}$ has a very weak luminescent fluorescence, suggesting that the PL band of $o$-PDCDs from $574 \mathrm{~nm}$ emerges as a consequence of the successful surface passivation of carbon nanoparticles with benzimidazole-containing oligomers of $o$-PD. Vedamalai et al. reported in a recent publication the preparation of $\mathrm{CDs}$ containing amino/hydroxyl functional groups on their surfaces, through a hydrothermal route using $o$-PD, that exhibit PL at $567 \mathrm{~nm}$ with the highest intensity for excitation at $420 \mathrm{~nm}^{25}$ Also, Jiang et $a l$. reported photoluminescent $o$-PD-CDs obtained from solvothermal treatment of 1,2-phenylenediamine exhibiting PL at $535 \mathrm{~nm}$ at the optimal excitation wavelength of $420 \mathrm{~nm} .{ }^{24}$ Figure 4 (b) presents the similarities between the normalized $\mathrm{PL}$ spectra of $o-\mathrm{PD}-\mathrm{CDs}$ and $o-\mathrm{PD}_{0}$ at the three most significant excitation wavelengths in the UV (i.e. $243 \mathrm{~nm}, 290 \mathrm{~nm}$ and $300 \mathrm{~nm}$ ). Despite some small differences between the two samples, we can presume that the PL detected in the UV region arises from the chemical products, similar to $o-\mathrm{PD}_{0}$, obtained along with $o$-PD-CDs in the process of carbon nanoparticles's surface passivation with $o$-PD. In order to confirm these assumptions we also recorded the excitation spectra of $o$-PD-CDs and $o-\mathrm{PD}_{0}$ at different $\mathrm{PL}$ wavelengths. As indicated in Figure 5 there are some obvious differences between the excitation spectra of the two samples.

As expected, in the case of $o-\mathrm{PD}_{0}$, the excitation spectrum retraces the features of the absorption spectrum regardless the PL emission wavelength. However, for $o-\mathrm{PD}-\mathrm{CDs}$, we obtained a distinct excitation spectrum for each of the selected PL wavelength. In particular, the feature of the spectra corresponding to the PL in the 366-425 nm (Figure 5 - red, orange and green curves) suggests that the emission in the UV originates from the CDs-free photoluminescent polymeric species found in the CDs's solution, while the band at $420 \mathrm{~nm}$ in the excitation spectrum recorded at $575 \mathrm{~nm}$ (Figure 5 - solid blue curve) clearly proves that the synthesized surface passivated $o$-PD-CDs exhibit a characteristic PL signal.

The quantum yield of the synthesized $o$-PDCDs, measured using fluorescent standard, was found to be $8 \%$, comparable to or even higher than the values reported in literature for passivation of CDs with small diamines ${ }^{9,26}$.

Time-resolved PL curves for $o$-PD-CDs and $o$ $\mathrm{PD}_{0}$ measured at $375 \mathrm{~nm}$ excitation are presented in Figure 6, while the obtained PL lifetime values are listed in Table 1.

The decay curve of $o$-PD-CDs was best fitted using double-exponential function. As shown in Table 1, o-PD-CDs exhibit a short dominant lifetime component of $1.6 \mathrm{~ns}(92.4 \%)$ and a longer one of $5.9 \mathrm{~ns}(7.6 \%)$, yielding an average lifetime of $1.93 \mathrm{~ns}$, similar to that of CDs prepared previously via different route. ${ }^{25}$

The bi-exponential behavior suggests the implication of two states in the photoluminescence process that are supposed to correspond to the energy gap and surface state transitions. ${ }^{27}$ It is believed that when excitation energy exceeds the energy gap, emission from the energy gap transition plays a leading role. However, when the excitation energy is lower than the energy gap, surface state transitions participate to the excitation-dependent behavior. Hence, the short lifetime component may correspond to the exciton emission, while the long component may be proof of the involvement of surface trapped states. ${ }^{27} \mathrm{CDs}$ with few nanosecond regime lifetimes could be exploited in optoelectronic and biological applications $^{28}$ while those with long PL lifetime could be used as convincing labels for long-term cell imaging, ${ }^{29,30}$ or have potential applicability in fluorescence lifetime imaging microscopy $(\text { FLIM })^{30}$ or solar cells. ${ }^{31}$ 


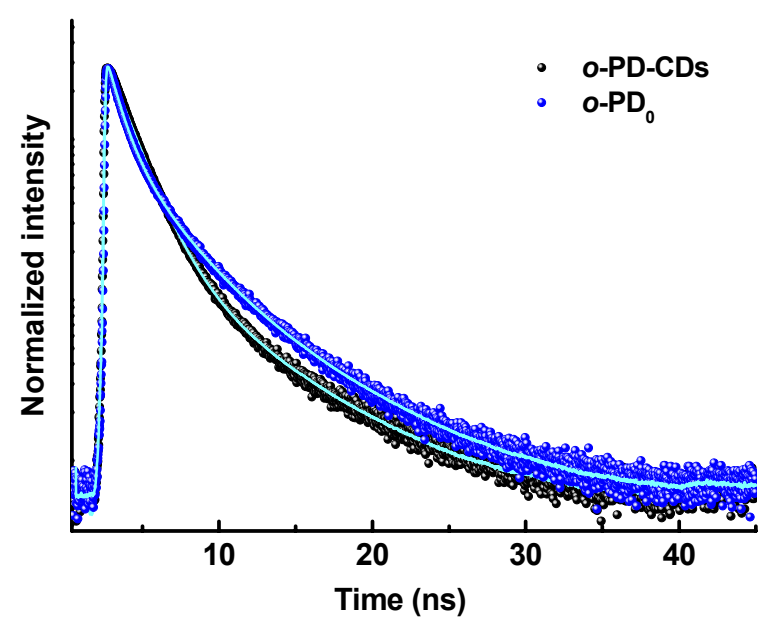

Fig. 6 - Fluorescence decay curves of $o$-PD-CDs and $o-\mathrm{PD}_{0}$ at $375 \mathrm{~nm}$ excitation, using $405 \mathrm{~nm}$ emission longpass filter (F1). Intensity is on logarithmic scale.

Table 1

Fluorescence decay parameters extracted for $o$-PD-CDs and $o-\mathrm{PD}_{0}$ (excitation at $375 \mathrm{~nm}$ )

\begin{tabular}{llllllll}
\hline Sample & $\begin{array}{l}\tau_{\mathbf{1}} \\
{[\mathbf{n s}]}\end{array}$ & $\begin{array}{l}\mathbf{A}_{\mathbf{1}} \\
{[\mathbf{\%}]}\end{array}$ & $\begin{array}{l}\tau_{\mathbf{2}} \\
{[\mathbf{n s}]}\end{array}$ & $\begin{array}{l}\mathbf{A}_{\mathbf{2}} \\
{[\mathbf{\%}]}\end{array}$ & $\begin{array}{l}\tau_{\mathbf{3}} \\
{[\mathbf{n s}]}\end{array}$ & $\begin{array}{l}\mathbf{A}_{\mathbf{3}} \\
{[\mathbf{\%}]}\end{array}$ & $\begin{array}{l}\tau_{\text {amp }} \\
{[\mathbf{n s}]}\end{array}$ \\
\hline \hline $\boldsymbol{o - P D - C D s}$ & $5.9 \pm 0.03$ & $7.6 \pm 0.01$ & $1.6 \pm 0.01$ & $92.4 \pm 0.4$ & - & - & 1.93 \\
\hline $\boldsymbol{o - P D}$ & $6.8 \pm 0.1$ & $8 \pm 0.6$ & $2.6 \pm 0.06$ & $30.3 \pm 1.2$ & $0.8 \pm 0.01$ & $61.7 \pm 2$ & 1.8 \\
\hline
\end{tabular}

$\tau_{\mathrm{n}}$ and $\mathrm{A}_{\mathrm{n}}$ is the lifetime and amplitude of the $\mathrm{n}^{\text {th }}$ component; $\tau_{\text {avg }}$ is the amplitude-weighted average lifetime calculated

with: $\tau_{\text {avg }}=\frac{\sum \tau_{i}^{*} A_{i}}{\sum A_{i}}$;

The fluorescence lifetime decay of the material obtained from the synthesis performed in the absence of carbon nanoparticles, $o-\mathrm{PD}_{0}$, displays a three-exponential behavior however the lifetime components and amplitudes are different from those extracted for $o$-PD-CDs (see Table 1) confirming the successful passivation towards the formation of completely new fluorescent products, i.e. CDs, with well established PL features.

\section{EXPERIMENTAL}

\section{Materials}

The carbon nanopowder $(<50 \mathrm{~nm}$, purity $99+\%)$, 1,2phenylenediamine $(o-\mathrm{PD})$ were purchased from SigmaAldrich. N,N-Dimethylformamide (DMF) (purchased from Sigma-Aldrich) was dried over calcium hydride and distilled under argon prior to use. Nitric acid (65\%) and thionyl chloride were obtained from Merck Millipore. Dialysis membrane tubing (cutoff molecular weight $1 \mathrm{kD}$ ) was supplied by Spectrum Laboratories. Water was deionized and purified by being passed through a Milli-Q water purification system.

\section{General procedure for carbon nanoparticles}

A commercially supplied carbon nanopowder sample (2g) was refluxed in an aqueous nitric acid $(2.6 \mathrm{M}, 160 \mathrm{~mL})$ for 18h. After being cooled to room temperature, the suspension was dialyzed against fresh water, and then centrifugated at $1000 \mathrm{~g}$ in order to retain the supernatant, from which surfaceoxidized carbon nanoparticles were recovered (about $150 \mathrm{mg}$ ).

\section{Functionalization of carbon nanoparticles with 1,2-phenylenediamine}

The obtained surface-oxidized carbon nanoparticles $(50 \mathrm{mg})$ were refluxed for $15 \mathrm{~h}$ in neat $\mathrm{SOCl}_{2}(5 \mathrm{~mL})$. The excess thionyl chloride was removed under reduced pressure and the recovered carbon nanoparticles were dispersed in anhydrous DMF $(1 \mathrm{~mL})$. To the suspension $o$-PD $(500 \mathrm{mg})$ was added and the reaction mixture was stirred vigorously under argon atmosphere for 3 days at $160^{\circ} \mathrm{C}$. The mixture was cooled to room temperature, dispersed in water $(30 \mathrm{~mL})$ via sonication and then centrifuged to $14000 \mathrm{rpm}$ when the supernatant was retained. The recovered supernatant was fractionated on a gel column (Sephadex G-100) to harvest the most fluorescent fraction, further referred to as $o$-PD-CDs.

Similar procedures have been carried out in the absence of carbon nanoparticles yielding $o-\mathrm{PD}_{0}$ for reference: $o$-PD $(400 \mathrm{mg})$ was dispersed in anhydrous DMF $(1 \mathrm{ml})$ and the reaction mixture was stirred vigorously under argon atmosphere for 3 days at $160^{\circ} \mathrm{C}$. The mixture was cooled to room temperature, dispersed in water $(30 \mathrm{~mL})$ via sonication and then centrifuged to $14000 \mathrm{rpm}$ when the supernatant was retained. The recovered supernatant was lyophilized to obtain $90 \mathrm{mg}$ of black powder, further referred to as $o-\mathrm{PD}_{0}$. 


\section{Measurements}

UV-Vis absorption spectra were performed at room temperature, in aqueous solution, using a Jasco V-670 UVVis-NIR spectrophotometer with a band width of $2 \mathrm{~nm}$ and $1 \mathrm{~nm}$ spectral resolution. Fourier transform infrared absorption (FT-IR) measurements were recorded with a Bruker Equinox 55 Fourier transform infrared spectrometer on solid samples. Each spectrum was measured in attenuated total reflectance (ATR) mode with 60 scans and $2 \mathrm{~cm}^{-1}$ resolution. Nuclear Magnetic Resonance (NMR) spectra were recorded in $\mathrm{D}_{2} \mathrm{O}$ as solvent at room temperature, on instruments operating at 600 $\mathrm{MHz}$ for ${ }^{1} \mathrm{H}$ and $150 \mathrm{MHz}$ for ${ }^{13} \mathrm{C}$, respectively. Chemical shifts $(\delta)$ are reported in parts per million (ppm) using residual solvent peak as internal reference. The steady-state photoluminescence analysis of CDs colloidal solutions was carried out using a Jasco LP-6500 spectrofluorimeter equipped with Xenon lamp as a light source. All photoluminescence spectra were corrected for the intensity of the excitation lamp and the wavelength dependence of the detection system. Fluorescence lifetime measurements of the analyzed solutions were performed on a PicoQuantMicroTime 200 time-resolved confocal fluorescence microscope system based on an inverted microscope (IX 71, Olympus) equipped with a UPLSAPO 60x/NA 1.2 water immersion objective. The excitation beam was provided by $8 \mu \mathrm{W}$ picosecond diode laser head (LDH-DC-375, Pico-Quant) operating at $375 \mathrm{~nm}$ and pulsed at 40 $\mathrm{MHz}$ repetition rate. The signal collected through the objective was spatially and spectrally filtered by a $50 \mu \mathrm{m}$ pinhole and emission longpass filter (HQ405LP, Chroma, Brattleboro, USA), respectively, before being focused on a PDM Single Photon Avalanche Diode (SPAD) from MPD. The detector signals were processed by the PicoHarp 300 Time-Correlated Single Photon Counting (TCSPC) data acquisition unit, from PicoQuant. Time-resolved fluorescence decay curves were recorded and analyzed using the SymPhoTime software (version 1.6) provided by PicoQuant employing a nonlinear iterative deconvolution algorithm for estimation of the fluorescence lifetimes. The instrument response function (IRF) was recorded from the laser light back scattered from plain cover glass working in similar experimental conditions. The relative quantum yields $(\mathrm{Q})$ of $o$-PD-CDs was measured by using Rhodamine $6 \mathrm{G}$ in ethanol as fluorescence standard, with the following equation: ${ }^{18}$

$$
Q=Q_{R} \frac{1}{I_{R}} \frac{A_{R}}{A} \frac{n^{2}}{n_{R}^{2}}
$$

where I is the integrated photoluminescence intensity, A is the absorbance at the excitation wavelength, $\eta$ is refractive index of the solvent and the subscript $\mathrm{R}$ denotes standard references.

\section{CONCLUSIONS}

In summary, new photoluminescent CDs were prepared from carboxylated carbon nanoparticles via reaction with 1,2-phenylenediamine in DMF as solvent. The functional groups at the surface of the photoluminescent CDs were studied by FT-IR and NMR methods while their photoluminescence properties were investigated through steady state and time-resolved fluorescence measurements. Structural and spectroscopic investigations re- vealed the formation of carbon dots passivated with benzimidazole-containing functionalities exhibiting photoluminescence quantum yield of $8 \%$ at $575 \mathrm{~nm}$ and lifetime of $1.9 \mathrm{~ns}$. These intriguing properties and advantageous surface chemistry of the synthesized carbon dots could open the door for new applications.

Acknowledgements. This work was supported by a grant from the Roumanian National Authority for Scientific Research, CNCS-UEFISCDI, project number PNII-ID-PCCE2011-2-0069.

\section{REFERENCES}

1. D. Jariwala, V. K. Sangwan, L. J. Lauhon, T. J. Marks and M. C. Hersam, Chem. Soc. Rev., 2013, 42, 28242860 .

2. L. Li and T. Dong, J. Mater. Chem. C, 2018, 6, 7944-7970.

3. A. Sachdev, I. Matai and P. Gopinath, RSC Adv., 2014, 4, 20915-20921.

4. X. Zhai, P. Zhang, C. Liu, T. Bai, W. Li, L. Dai and W. Liu, Chem. Commun., 2012, 48, 7955-7957.

5. A. Diac, M. Focsan, C. Socaci, A.-M. Gabudean, C. Farcau, D. Maniu, E. Vasile, A. Terec, L. M. Veca and S. Astilean, RSC Adv., 2015, 5, 77662-77669.

6. Y.-P. Sun, B. Zhou, Y. Lin, W. Wang, K. A. S. Fernando, P. Pathak, M. J. Meziani, B. A. Harruff, X. Wang, H. Wang, P. G. Luo, H. Yang, M. E. Kose, B. Chen, L. M. Veca and S.-Y. Xie, J. Am. Chem. Soc., 2006, 128, 77567757.

7. H. Ding, L.-W. Cheng, Y.-Y. Ma, J.-L. Kong and H.-M. Xiong, New J. Chem., 2013, 37, 2515-2520.

8. C. Wang, Z. Xu and C. Zhang, ChemNanoMat, 2015, 1, $122-127$.

9. J. Zhou, P. Lin, J. Ma, X. Shan, H. Feng, C. Chen, J. Chen and Z. Qian, RSC Adv., 2013, 3, 9625-9628.

10. G. E. LeCroy, S. K. Sonkar, F. Yang, L. M. Veca, P. Wang, K. N. Tackett, J.-J. Yu, E. Vasile, H. Qian, Y. Liu, P. (George) Luo and Y.-P. Sun, ACS Nano, 2014, 8, $4522-4529$.

11. H. Nie, M. Li, Q. Li, S. Liang, Y. Tan, L. Sheng, W. Shi and S. X.-A. Zhang, Chem. Mater., 2014, 26, 3104-3112.

12. A.-M. Craciun, A. Diac, M. Focsan, C. Socaci, K. Magyari, D. Maniu, I. Mihalache, L. M. Veca, S. Astilean and A. Terec, RSC Adv., 2016, 6, 56944-56951.

13. C. Liu, R. Wang, B. Wang, Z. Deng, Y. Jin, Y. Kang and J. Chen, Microchimica Acta, 2018, 185, 539.

14. L. Song, Y. Cui, C. Zhang, Z. Hu and X. Liu, RSC Adv., 2016, 6, 17704-17712

15. H. Nie, M. Li, S. Liang, Y. Tan, L. Sheng, W. Shi and S.X.-A. Zhang, Chem. Mater. 2014, 26, 3104-3112

16. a) P. K. Singh and O. Silakari "Benzimidazole: Journey From Single Targeting to Multitargeting Molecule" in "Key Heterocycle Cores for Designing Multitargeting Molecules", Elsevier, 2018, p. 31-52; b) Y.K. Yoon, T.S. Chia, C.K. Quah, W.L. Lim, C.W. Oo, A.N. Shirazi, K. Parang and T.S. Choon, Lett. Org. Chem., 2017, 14, 33-38.

17. a) M. Mamada, C. Perez-Bolivar, D. Kumaki, N.A. Esipenko, S. Tokito and P. Anzenbacher Jr., Chem. Eur $J ., 2014,20,11835-11846$; b) E.G. Cansu-Ergun, Polym. Rev., 2018, 58, 42-62; c) B. Jedrzejewska, P. Krawczyk, 
M. Gordel and M. Samoc, Dyes Pigments, 2014, 111, 162-175.

18. P.P. Kattimani, R. R. Kamble and G. Y. Meti, $R S C A d v$, 2015, 5, 29447-29455.

19. a) H. Mostafavi, M. R. Islami, E. Ghonchepour and A. M. Tikdari, Chem. Pap., 2018, 72, 2973-2978; b) J. Zhu, Z. Zhang, C. Miao, W. Liu and W. Sun, Tetrahedron, 2017, 73, 3458-3462.

20. F. Wang, S. Pang, L. Wang, Q. Li, M. Kreiter and C. Liu, Chem. Mater., 2010, 22, 4528-4530.

21. N. Dhenadhayalan and K.-C. Lin, Sci. Rep., 2015, 5, 10012.

22. K. J. Morgan, J. Chem. Soc., 1961, 2343-2347.

23. B. Wang, S. Wang, Y. Wang, Y. Lv, H. Wu, X. Ma and M. Tan, Biotechnol. Lett., 2016, 38, 191-201.

24. K. Jiang, S. Sun, L. Zhang, Y. Lu, A. Wu, C. Cai and H. Lin, Angew. Chem. Int. Ed., 2015, 54, 5360-5363.
25. M. Vedamalai, A. P. Periasamy, C.-W. Wang, Y.-T. Tseng, L.-C. Ho, C.-C. Shih and H.-T. Chang, Nanoscale, 2014, 6, 13119-13125.

26. W. Dong, Y. Dong, Y. Wang, S. Zhou, X. Ge, L. Sui and J. Wang, Spectrochim. Acta. A. Mol. Biomol. Spectrosc., 2013, 116, 209-213.

27. X. Li, S. Zhang, S. A. Kulinich, Y. Liu and H. Zeng, Sci. Rep., 2014, 4, 4976.

28. D. Sun, R. Ban, P.-H. Zhang, G.-H. Wu, J.-R. Zhang and J.-J. Zhu, Carbon, 2013, 64, 424-434.

29. J. K. Jaiswal, H. Mattoussi, J. M. Mauro and S. M. Simon, Nat. Biotechnol., 2003, 21, 47-51.

30. J.-H. Liu, S.-T. Yang, X.-X. Chen and H. Wang, Curr. Drug Metab., 2012, 13, 1046-1056.

31. R. Narayanan, M. Deepa and A. K. Srivastava, J. Mater. Chem. A, 2013, 1, 3907-3918. 